
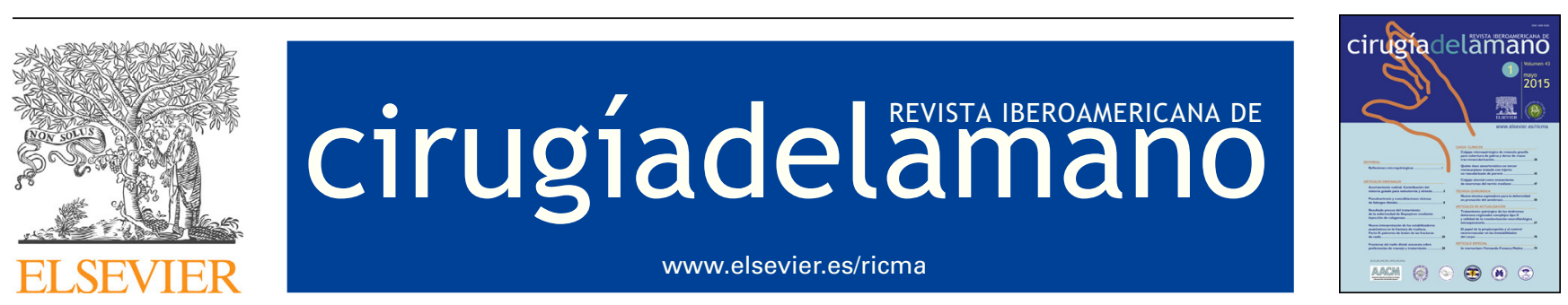

ARTÍCULO ORIGINAL

\title{
Análisis artroscópico de las lesiones asociadas a las fracturas de escafoides carpiano
}

\author{
V. Carratalá Baixauli*, F. Lucas García, E. Sánchez Alepuz y R. Calero Ferrandiz
}

Servicio de Cirugía Ortopédica y Traumatología, Unión de Mutuas, Valencia, España

Recibido el 15 de junio de 2015; aceptado el 29 de agosto de 2015

Disponible en Internet el 28 de septiembre de 2015

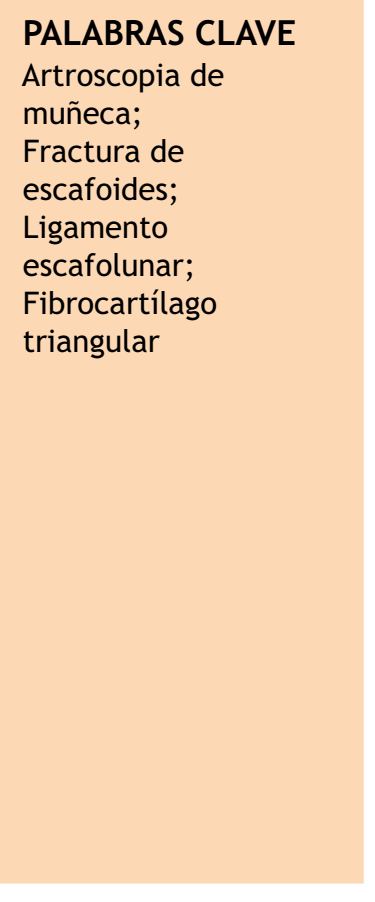

\begin{abstract}
Resumen
Objetivo: La artroscopia de muñeca ha permitido el diagnóstico y el tratamiento de las fracturas a nivel de la muñeca, así como de las lesiones asociadas a estas.

Existen lesiones asociadas a las fracturas de escafoides que pueden condicionar el éxito del tratamiento y llevarnos a resultados poco satisfactorios y a un retraso en la recuperación funcional.

Material y método: Se estudiaron y cuantificaron las lesiones asociadas encontradas en 17 casos con fractura aguda de cintura de escafoides que fueron tratadas mediante reducción y colocación artroscópica de un tornillo canulado de escafoides. Se analizaron los resultados del tratamiento con la escala de muñeca de la Clínica Mayo (Mayo Wrist Score) y la consolidación con radiografías simples.

Resultados: Se diagnosticaron lesiones asociadas de tejidos blandos y/o condrales/osteocondrales en 14 casos, lo que supone un $82 \%$ de los casos. Las lesiones más frecuentemente encontradas fueron las lesiones del ligamento escafolunar (53\%) en diferentes grados, las lesiones del fibrocartílago triangular $(35,3 \%$ ) y las lesiones osteocondrales $(35,3 \%)$. Los resultados funcionales (Mayo Wrist Score) fueron excelentes o buenos en 13 de los pacientes (76,5\%), en 3 casos $(17,5 \%)$ los resultados fueron regulares y solo un caso $(5,9 \%)$ tuvo un pobre resultado.

Conclusiones: La artroscopia ofrece una valoración completa de la muñeca y consigue diagnosticar lesiones asociadas a las fracturas de cuello de escafoides que pueden condicionar el resultado final del tratamiento.

๑ 2015 SECMA. Publicado por Elsevier España, S.L.U. Este es un artículo Open Access bajo la licencia CC BY-NC-ND (http://creativecommons.org/licenses/by-nc-nd/4.0/).
\end{abstract}

\footnotetext{
* Autor para correspondencia.

Correo electrónico: vteca@ono.com (V. Carratalá Baixauli).
} 


\section{KEYWORDS}

Wrist arthroscopy; Scaphoid fracture; Scapholunate ligament; Triangular fibrocartilage complex

\section{Arthroscopic analysis of injuries associated with carpal scaphoid fractures}

\begin{abstract}
Objective: Arthroscopy has enabled wrist fractures and the injuries associated with them to be diagnosed and treated. There are injuries associated with scaphoid fractures, which may influence the success of treatment and can lead to an unsatisfactory result and delay in the functional recovery.

Material and method: A prospective study was conducted on 17 cases with acute scaphoid body fractures treated by arthroscopic reduction and placement of a cannulated scaphoid screw. Systematic arthroscopy evaluation (radiocarpal and midcarpal) was performed, recording the presence or absence of associated lesions in each case. Treatment outcome was measured using the Mayo Wrist Score, and the fracture consolidation evaluated with plain x-rays.

Results: Associated soft tissues injuries and/or chondral/osteochondral injuries were diagnosed in $14(82 \%)$ cases. The most frequently found lesions were scapholunate ligament injuries (53\%) in different degrees, triangular fibrocartilage complex (TFCC) lesions (35.3\%), and osteochondral lesions (35.3\%). Functional outcomes (Mayo Wrist Score) were excellent or good in 13 patients $(76.5 \%)$, and in 3 cases $(17.5 \%)$ the results were fair, and only 1 case $(5.9 \%)$ had a poor outcome.

Conclusions: Wrist arthroscopy is a valuable tool for the diagnosis and treatment of wrist injuries. There are associated injuries in acute scaphoid body fractures that could influence treatment outcomes.

(C) 2015 SECMA. Published by Elsevier España, S.L.U. This is an open access article under the CC BY-NC-ND license (http://creativecommons.org/licenses/by-nc-nd/4.0/).
\end{abstract}

\section{Introducción}

Aunque clásicamente las fracturas de escafoides han sido tratadas conservadoramente, siendo este un tratamiento eficaz ${ }^{1,2}$, en muchos casos la inmovilización prolongada comportaba las consiguientes complicaciones, sobre todo de rigidez, pérdida de fuerza y retraso en la reincorporación laboral y/o deportiva ${ }^{3}$. Además, este tratamiento mediante inmovilización también se ha relacionado con tasas de seudoartrosis de entre un 5 y un 15\%, principalmente en las fracturas con mayor desplazamiento ${ }^{1,4,5}$.

La introducción de la fijación interna para las fracturas de escafoides por medio de técnicas poco invasivas o percutáneas $^{6}$ permitió una mejor estabilización de la fractura y un inicio de la movilidad y una reincorporación laboral $y / 0$ deportiva más precoces ${ }^{7-10}$, así como unos porcentajes menores de no unión, siendo la tasa de seudoartrosis, según los estudios, de entre un 1 y un $5 \%^{8,11}$, Sin embargo, existen lesiones asociadas a las fracturas de escafoides que pueden condicionar el buen resultado del tratamiento y que pueden, más allá de la consolidación de la fractura, retrasar la curación y llevarnos a un resultado global no satisfactorio ${ }^{12,13}$. El objetivo de este trabajo es conocer las lesiones asociadas a las fracturas agudas de la cintura del escafoides mediante la valoración artroscópica tras la reducción y colocación de un tornillo canulado de escafoides. Además, se ha analizado el resultado del tratamiento haciendo un seguimiento de los pacientes hasta la consolidación de la fractura y el alta, cuantificando los resultados con la escala de muñeca de la Clínica Mayo (Mayo Wrist Score $^{14}$ ) y la consolidación con radiografías simples (figs. 1-5).

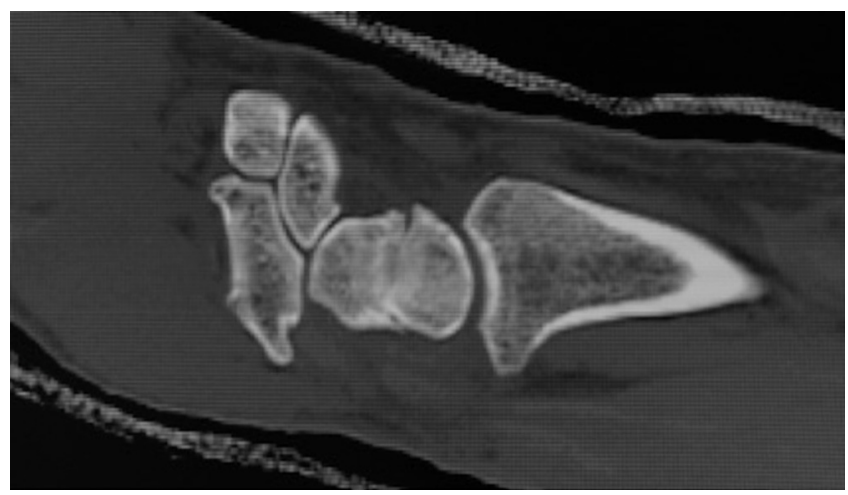

Figura 1 TAC. Fractura de cintura de escafoides.

\section{Material y método}

Para realizar el estudio se revisaron los datos obtenidos del tratamiento artroscópico de 17 casos asistidos en nuestro centro entre los años 2012 y 2014 , que presentaban fracturas de escafoides.

Los criterios de inclusión en el estudio se establecieron para casos de fracturas agudas (menos de 3 semanas de evolución) de cintura de escafoides con cualquier grado de desplazamiento, por ser las fracturas de escafoides más frecuentes y tener un tratamiento más estandarizado.

Se excluyeron del estudio las fracturas de polo proximal y del polo distal, los pacientes con lesiones traumáticas previas o con antecedentes de infección en la muñeca, las fracturas abiertas y los pacientes con contraindicación para la cirugía. 


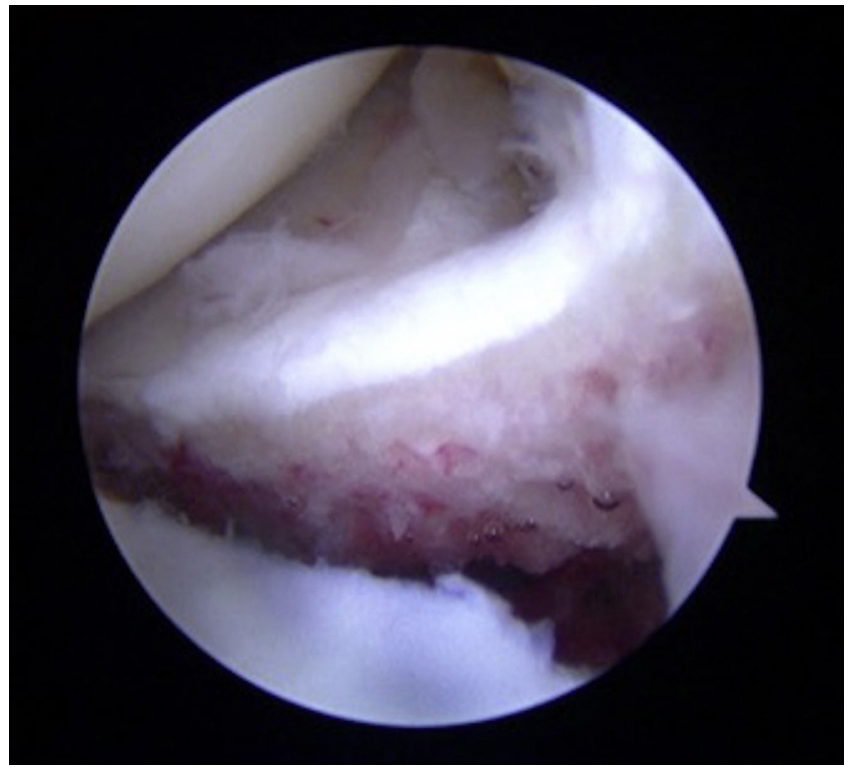

Figura 2 Fractura desplazada de escafoides vista desde espacio mediocarpiano (portal ulnar).

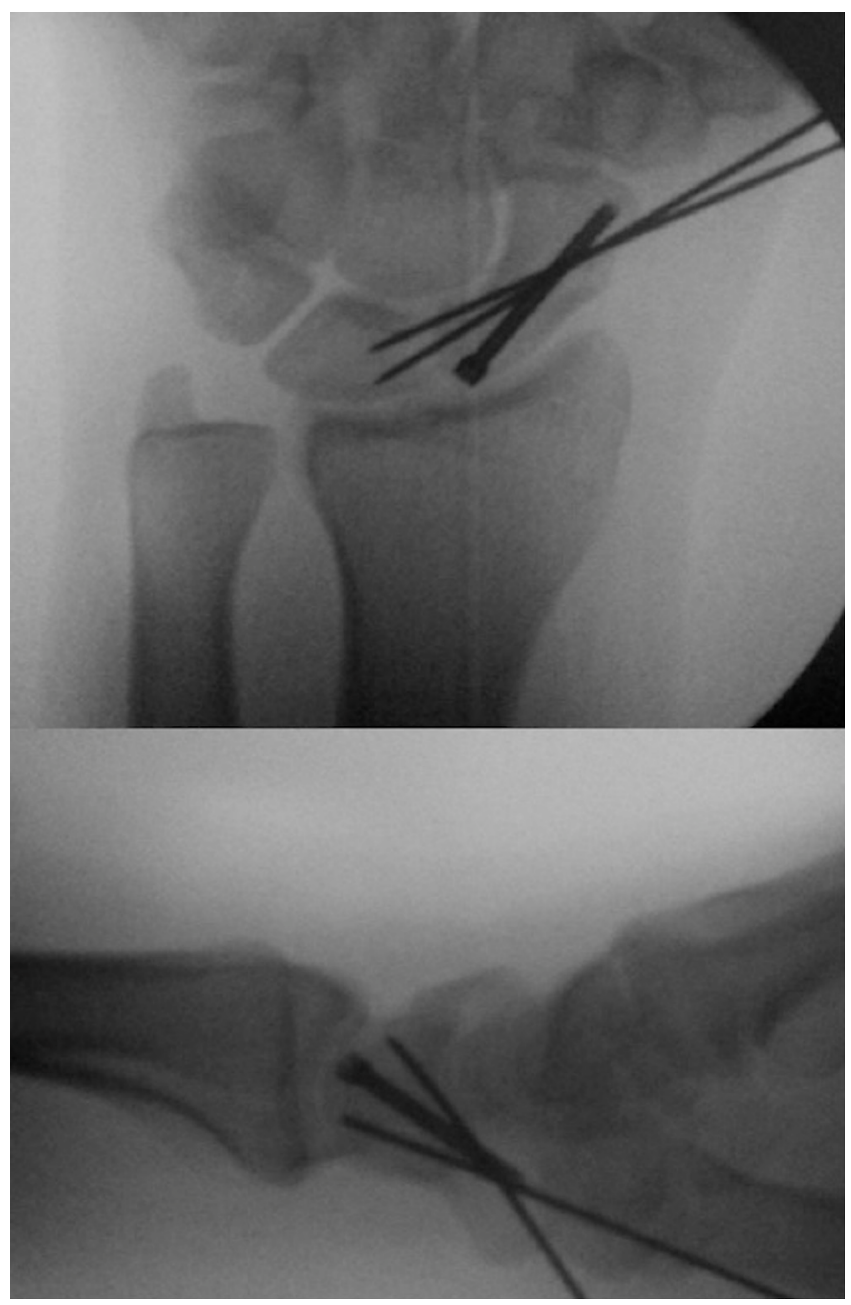

Figura 3 Resultado final de una fractura asociada a lesión del ligamento escafolunar.
La mayoría de los pacientes eran varones con una relación de 15/2. La edad media se situó en 40 años (rango 26-58 años), siendo la mediana 37 años. La muñeca izquierda se vio afectada en 8 pacientes frente a las 9 lesiones en muñecas derechas.

El diagnóstico se realizó mediante radiografías simples de muñeca, completándose el estudio con imágenes de tomografía computarizada (fig. 1).

Se clasificaron las lesiones de los ligamentos escafolunar (EL) y lunotriquetral (LT) según la clasificación de Geissler y Freeland ${ }^{15}$, y las lesiones del complejo fibrocartílago triangular del carpo (CFCT) según la clasificación de Palmer ${ }^{16}$.

\section{Técnica artroscópica}

Las cirugías fueron realizadas por el mismo cirujano, bajo anestesia regional del brazo afectado. Se empleó un sistema de tracción (Acumed, Hillsboro, EE. UU.), con una tracción de 10-15 libras y una flexión de muñeca de 20-30。. No se empleó sistema de irrigación con bomba. Los portales utilizados para la artroscopia de muñeca fueron los portales estándar 3-4, 4-5, $6 \mathrm{R}$, portal mediocarpiano radial y portal mediocarpiano ulnar. Los portales mediocarpianos se emplearon tanto para la reducción de las fracturas, como para la evaluación de las lesiones asociadas.

Durante la artroscopia se realizó la reducción directa de la fractura (fig. 2) y se empleó para la fijación un tornillo canulado de 2,4 mm HCS (Synthes, Paoli, EE. UU.), colocado en dirección de dorsal a volar desde el portal 3-4 y con visión artroscópica directa desde el portal 6R según técnica descrita por Geissler ${ }^{17}$. Se empleó un intensificador de imágenes intraoperatoriamente para comprobar la correcta longitud y colocación del tornillo.

Posteriormente, se realizó una revisión sistemática de las lesiones tanto en el espacio radiocarpiano como en el mediocarpiano, que fueron registradas en todos los casos. Se exploraron los ligamentos intrínsecos y extrínsecos, las superficies condrales de ambos espacios articulares y las lesiones capsulares.

En espacio radiocarpiano se exploraron los ligamentos intrínsecos EL y LT, y los extrínsecos, radioescafocapitate, radiolunar largo y corto, radioescafolunar de Testut, ulnolunar y ulnotriquetral, y la cápsula dorsal con el ligamento radiocarpiano dorsal. También se realizó la exploración del CFCT y las superficies articulares distal del radio y proximal de la primera fila del carpo clasificando todas las lesiones localizadas.

En el espacio mediocarpiano se exploraron de nuevo los ligamentos EL y LT, la continuación del ligamento radioescafocapitate y de los ulnocarpianos, las superficies condrales de ambas hileras del carpo y los ligamentos volares.

Las lesiones de los ligamentos intrínsecos EL y LT fueron tratadas mediante desbridamiento e inmovilización, añadiendo fijación con agujas de Kirschner desde el escafoides al semilunar en los grados III y IV de Geissler (fig. 3). Las lesiones del ligamento EL y del LT grados ıy ॥ llevaron una férula antebraquial durante 4 semanas y aquellas tratadas con agujas de Kirschner fueron inmovilizadas durante 6 semanas, tras las cuales se retiraron la férula y las agujas. Las lesiones del CFCT con avulsión capsular ulnar 1B de Palmer fueron suturadas con técnica fuera a dentro con PDS de 


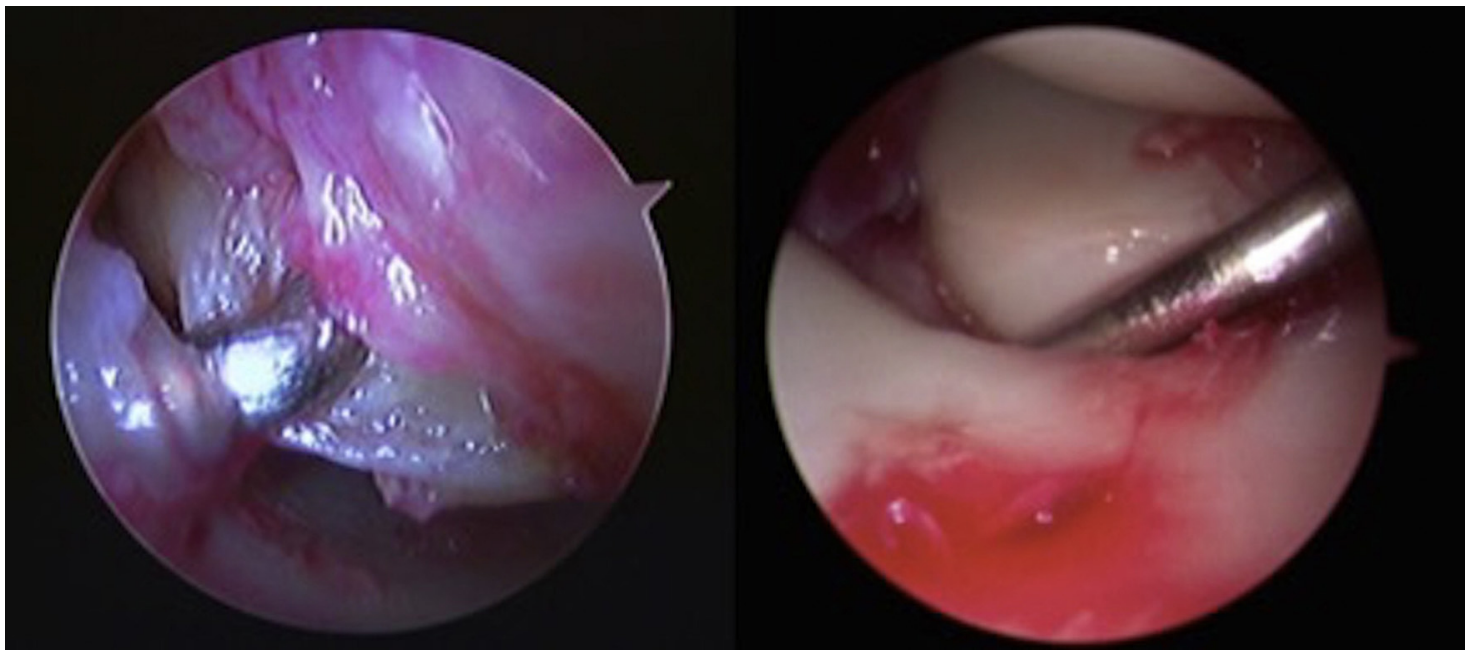

Figura 4 Lesión del ligamento escafolunar de grado III desde el espacio mediocarpiano y radiocarpiano.

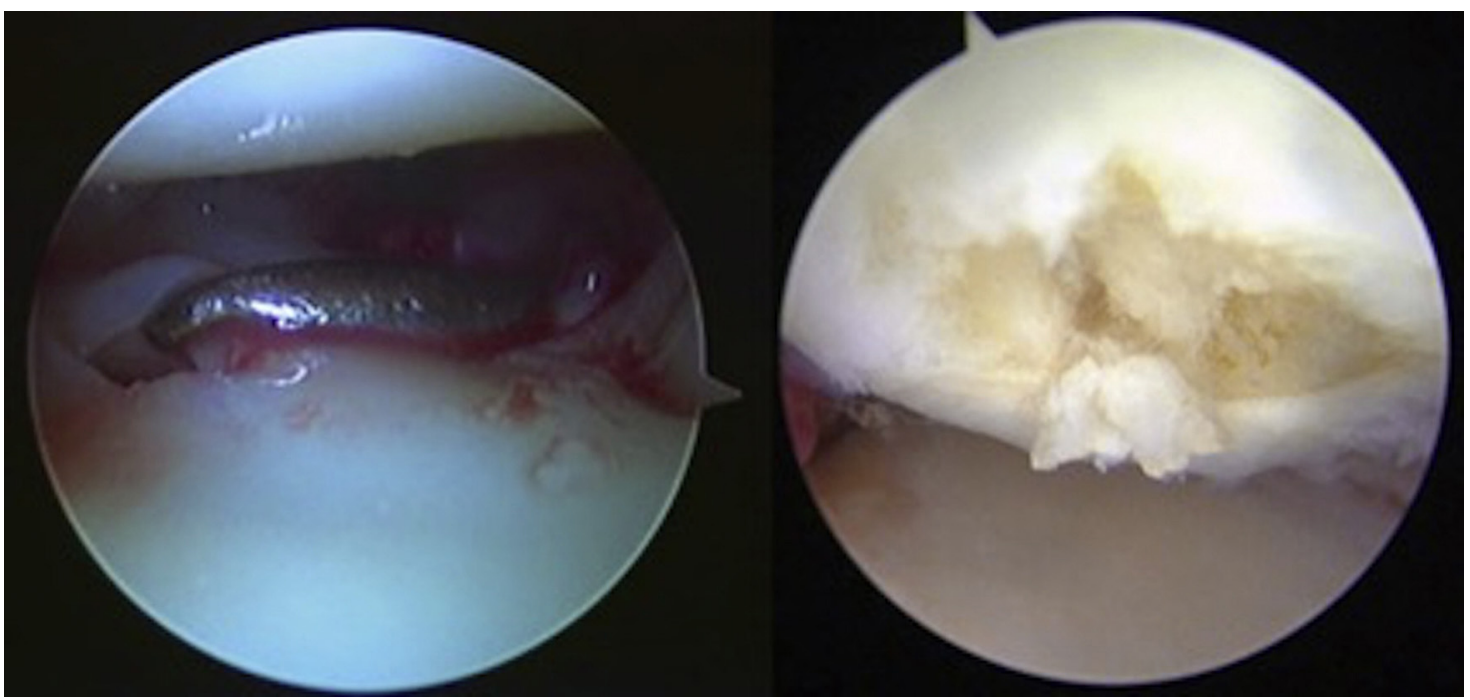

Figura 5 Lesión 1A del CFCT y lesión osteocondral en hueso grande.

$3 / 0$, mientras que las lesiones centrales $1 \mathrm{~A}$ de Palmer fueron desbridadas hasta conseguir un margen estable. Las reparaciones del CFCT llevaron una férula braquial en supinación durante 4 semanas y una férula antebraquial 2 semanas más.

En las lesiones condrales encontradas fueron extraídos los restos libres intraarticulares y se limpió la superficie articular. En un caso se realizaron perforaciones y microfracturas en hueso subcondral, siendo inmovilizado con una férula después de la cirugía durante 3 semanas.

El resto de los casos en los que se hallaron lesiones asociadas fueron inmovilizados con una férula antebraquial durante 2 semanas tras la cirugía.

Todos los pacientes fueron remitidos a tratamiento rehabilitador tras la retirada de la inmovilización.

Durante el seguimiento, la valoración funcional de los todos los pacientes se realizó con la escala Mayo Wrist Score. La fuerza de prensión de puño se midió en kilogramos con dinamómetro manual JAMAR.

Se valoró el tiempo hasta la consolidación de la fractura y el tiempo hasta el alta laboral. El tiempo de seguimiento fue de 6 meses tras el alta laboral y/o deportiva del paciente, siendo la media de seguimiento unos 10 meses desde la fractura.

\section{Resultados}

Tras la artroscopia de muñeca realizada a los 17 pacientes con fractura de escafoides se diagnosticaron lesiones asociadas de tejidos blandos y/o condrales/osteocondrales en 14 casos $(82 \%)$. Las lesiones más frecuentemente encontradas fueron la lesión del ligamento EL (fig. 4) y la rotura del CFCT (fig. 5), presentes en 9/17 y 6/17 pacientes, respectivamente. Uno de los pacientes presentó lesiones en todos los ligamentos intrínsecos, además de lesiones condrales y rotura del CFCT. La distribución de las diversas lesiones se muestra en la tabla 1.

La mayor parte de las roturas de los ligamentos intrínsecos carpianos eran desgarros parciales, de grado I 0 ॥ según la clasificación de Geissler, hallándose estos grados 
Tabla 1 Lesiones asociadas encontradas en los pacientes durante la artroscopia de muñeca y resultados funcionales de los pacientes según la escala Mayo Wrist Score

\begin{tabular}{|c|c|c|c|c|c|c|c|c|c|c|}
\hline Paciente & $\begin{array}{l}\text { Edad, } \\
\text { años }\end{array}$ & Sexo & Lat & Lesión EL & Lesión LT & CFCT & $\begin{array}{l}\text { Lesión } \\
\text { condral }\end{array}$ & $\begin{array}{l}\text { Cápsula/ } \\
\text { lig. ext }\end{array}$ & $\begin{array}{l}\text { Días de } \\
\text { baja }\end{array}$ & $\begin{array}{l}\text { Resultados } \\
\text { funcionales }\end{array}$ \\
\hline Paciente 1 & 26 & V & Izd & No & No & Sí (C) & No & No & 92 & Excelente \\
\hline Paciente 2 & 38 & V & Izd & No & Sí (G2) & No & No & No & 112 & Excelente \\
\hline Paciente 3 & 35 & V & Der & Sí (G1) & No & No & No & No & 86 & Bueno \\
\hline Paciente 4 & 53 & V & Izd & Sí (G2) & No & Sí (CUB) & Sí (RE) & Sí (dorsal) & 173 & Regular \\
\hline Paciente 5 & 44 & V & Der & No & No & No & No & No & 64 & Excelente \\
\hline Paciente 6 & 36 & V & Izd & Sí (G2) & No & No & No & No & 98 & Bueno \\
\hline Paciente 7 & 47 & V & Der & No & No & No & No & No & 81 & Bueno \\
\hline Paciente 8 & 58 & V & Izd & Sí (G3) & Sí (G2) & Sí $(C+C U B)$ & Sí (RE) & No & 201 & Regular \\
\hline Paciente 9 & 42 & V & Izd & Sí (G4) & No & SI (C) & Sí (CAP + RE) & Sí (VOL MC) & 221 & Pobre \\
\hline Paciente 10 & 31 & $M$ & Der & No & No & No & No & No & 70 & Excelente \\
\hline Paciente 11 & 29 & V & Izd & No & Sí (G1) & No & No & No & 89 & Excelente \\
\hline Paciente 12 & 32 & V & Der & No & No & Sí (CUB) & Sí (RE) & $\begin{array}{l}\text { Sí (asociada } \\
\text { a fractura } \\
\text { de radio) }\end{array}$ & 157 & Bueno \\
\hline Paciente 13 & 48 & V & Der & Sí (G2) & No & No & No & No & 94 & Bueno \\
\hline Paciente 14 & 49 & V & Der & Sí (G3) & No & No & Sí (SL) & No & 141 & Bueno \\
\hline Paciente 15 & 31 & V & Der & Sí (G1) & No & No & No & No & 80 & Excelente \\
\hline Paciente 16 & 40 & V & Izd & No & No & Sí (CUB) & No & No & 87 & Bueno \\
\hline Paciente 17 & 47 & $M$ & Der & Sí (G1) & No & No & Sí (TR) & No & 123 & Regular \\
\hline
\end{tabular}

C: central 1A; CAP: capitate; CFCT: complejo del fibrocartílago triangular; CUB: cubital 1B; Der: derecho; EL: escafolunar; G: grados de Clasificación de Geissler; Izd: izquierdo; Lat: lateralidad; LT: lunotriquetral; M: mujer; RE: radioescafoidea; SL: semilunar; TR: trapecio; V: varón; VOL MC: volares mediocarpianos.

de rotura en 9 de los 12 pacientes que presentaban lesiones en los ligamentos intrínsecos carpianos. Los otros 3 pacientes presentaban lesiones completas de grado III y grado Iv. El paciente con lesiones de grado Iv,se corresponde con el paciente que presentaba mayor edad y la mayor parte de los tipos de lesiones. Las lesiones a nivel de CFCT se visualizaron tanto a nivel ulnar (en $3 / 6$ pacientes), como a nivel central ( $2 / 6$ pacientes), y en ambos niveles ( $1 / 6$ paciente). Tres pacientes presentaron roturas en la cápsula articular, que se trataron con desbridamiento e inmovilización temporal. Las lesiones condrales aparecieron en 6 de los pacientes, siendo el lugar más frecuente de lesión la fosa radioescafoidea que se encontraba lesionada en 4 de estos pacientes. Aparecieron además lesiones osteocondrales a nivel del hueso grande, escafoides, semilunar y trapecio (fig. 5).

De acuerdo con el Mayo Wrist Score, 13/17 de los pacientes $(76,5 \%)$ tuvieron excelentes o buenos resultados funcionales en la última visita de seguimiento, $3 / 17$ casos $(17,5 \%)$ tuvieron resultados regulares y solo $1 / 17$ caso $(5,9 \%)$ tuvo un pobre resultado (tabla 1). Los pacientes con los peores resultados correspondían con los pacientes que presentaban el mayor y más grave número de lesiones. Tres de los 4 pacientes presentaban lesiones de diversos grados en el ligamento EL, CFCT y lesiones condrales.

Todos los pacientes regresaron a sus actividades previas, incluyendo el trabajo y la práctica deportiva, tras una media de días de baja laboral de 116 días. Únicamente el paciente con el resultado pobre precisó la reorganización de su puesto de trabajo. Los pacientes con un mayor número de lesiones asociadas precisaron mayor número de días para su reincorporación laboral (tabla 1) y presentaron peores resultados funcionales. Únicamente un paciente con un resultado funcional bueno precisó un número mayor de días de baja que el resto debido a que asociaba una fractura de radio distal.

\section{Discusión}

Con la realización de este estudio hemos podido constatar la alta incidencia de lesiones ligamentosas y osteocondrales asociadas a las fracturas agudas del cuerpo del escafoides. En nuestra serie, 14 de los 17 pacientes tenían una lesión asociada, lo que suponía el $82 \%$ de los pacientes. La incidencia publicada previamente de lesiones asociadas de tejidos blandos relacionadas con las fracturas de escafoides oscilaron entre un mínimo del $35 \%^{18}$ y un máximo del $83 \%^{19}$ (tabla 2). Los porcentajes de lesiones asociadas a fracturas de escafoides publicados no difieren demasiado de los hallados en nuestro estudio. Jørgsholm et al. ${ }^{19}$ y Caloia et al. ${ }^{20}$ presentaron porcentajes similares al nuestro, con un 82,9 y un $62,5 \%$, respectivamente. La relación varón/mujer de nuestro trabajo $(15 / 2)$ coincide con la mayor incidencia en varones publicada en el resto de los estudios sobre fracturas de escafoides ${ }^{13}$.

Las lesiones parciales del ligamento EL y del CFCT fueron las lesiones de tejidos blandos más frecuentes en nuestro estudio, siendo encontradas en 9 y 6 pacientes, respectivamente. En términos porcentuales, las lesiones del ligamento EL suponían un 53\% y las lesiones del CFCT un 35,3\% de los pacientes, los cuales se asemejaban completamente a los resultados en el resto de estudios ${ }^{18-20}$. La mayoría de los estudios carecen de casos con roturas EL de grado iv de la clasificación de Geissler. Solamente Jørgsholm et al. ${ }^{19}$ 
Tabla 2 Resultados comparativos de lesiones asociadas a fracturas de escafoides en nuestra serie con el resto de estudios publicados

\begin{tabular}{|c|c|c|c|c|c|c|c|c|c|c|}
\hline & \multirow{2}{*}{\multicolumn{2}{|c|}{$\frac{\text { Carratala/Lucas }}{\text { N. }{ }^{\circ}}$}} & \multirow{2}{*}{\multicolumn{2}{|c|}{$\frac{\text { Caloia }}{\text { N. }{ }^{\circ}}$}} & \multirow{2}{*}{\multicolumn{2}{|c|}{$\frac{\text { Jørgsholm }}{\text { N. }{ }^{\circ}}$}} & \multirow{2}{*}{\multicolumn{2}{|c|}{$\frac{\text { Wong }}{\mathrm{N} .^{\circ}}$}} & \multirow{2}{*}{\multicolumn{2}{|c|}{$\begin{array}{l}\text { Shih } \\
\text { N. }{ }^{\circ}\end{array}$}} \\
\hline & & & & & & & & & & \\
\hline Pacientes & 17 & & 24 & & 41 & & 52 & & & \\
\hline Pacientes con lesiones & 14 & $82 \%$ & 15 & $63 \%$ & 34 & $83 \%$ & 18 & $35 \%$ & 15 & \\
\hline Lesión EL & 9 & $53 \%$ & 7 & $29 \%$ & 29 & $71 \%$ & 6 & $11 \%$ & 2 & $13,3 \%$ \\
\hline Grado I & 3 & $17,6 \%$ & 3 & $12,5 \%$ & 7 & $17 \%$ & & & & \\
\hline Grado II & 3 & $17,6 \%$ & 4 & $16 \%$ & 9 & $22 \%$ & & & & \\
\hline Grado III & 2 & $11,8 \%$ & 0 & 0 & 3 & $7 \%$ & & & & \\
\hline Grado IV & 1 & $5,9 \%$ & 0 & 0 & 10 & $24 \%$ & & & & \\
\hline Lesión LT & 3 & $17,6 \%$ & 4 & $17 \%$ & 8 & $20 \%$ & 10 & $19 \%$ & 4 & $26,7 \%$ \\
\hline Grado I & 1 & $5,9 \%$ & 2 & $8 \%$ & 4 & $10 \%$ & & & & \\
\hline Grado II & 2 & $11,8 \%$ & 2 & $8 \%$ & 2 & $5 \%$ & & & & \\
\hline Grado III & 0 & 0 & 0 & 0 & 2 & $5 \%$ & & & & \\
\hline Grado IV & 0 & 0 & 0 & 0 & 0 & 0 & & & & \\
\hline Lesión CFCT & 6 & $35,3 \%$ & 6 & $25 \%$ & 11 & $27 \%$ & 3 & $6 \%$ & 5 & $33 \%$ \\
\hline Lesión condral & 6 & $35,3 \%$ & 2 & $8 \%$ & & & & & 6 & $40 \%$ \\
\hline Lesión capsular & 3 & $17,6 \%$ & 2 & $8 \%$ & 25 & $61 \%$ & 1 & $2 \%$ & & \\
\hline Volar & 1 & $5,9 \%$ & 2 & $8 \%$ & 9 & $22 \%$ & 1 & $2 \%$ & & \\
\hline Dorsal & 1 & $5,9 \%$ & 0 & 0 & 16 & $39 \%$ & 0 & 0 & & \\
\hline Fractura de radio & 1 & $5,9 \%$ & 0 & 0 & 0 & 0 & 0 & 0 & & \\
\hline Edad media (rango) & $40,3(26-52)$ & $32(17-75)$ & $32(14-71)$ & & & & & & 29 & 48) \\
\hline
\end{tabular}

EL: escafolunar; LT: lunotriquetral; CFCT: complejo del fibrocartílago triangular.

presentaron una incidencia elevada de este grado de lesión con $10 / 41$ pacientes $(24 \%)$, lejos de $1 / 17(5,9 \%)$ registrado en nuestro estudio.

En cuanto a las roturas del CFCT, las hallamos en 6 de 17 pacientes (35\%), lo cual se asemeja considerablemente a los resultados de la bibliografía, donde se informa de lesión asociada del CFCT entre el 6 y el $33 \%$ de los pacientes ${ }^{13,18}$. Es posible que un análisis más detallado del tipo de lesiones del CFCT según la clasificación de Palmer nos podría aportar mayor información en relación con qué zona del CFCT es más propensa a lesión tras una fractura de escafoides. Las roturas del ligamento LT fueron las lesiones más inusuales, apareciendo en nuestra serie en 3 de 17 pacientes $(17,6 \%)$. Nuestros resultados son comparables con otros estudios, donde se encontraron lesiones asociadas del ligamento LT entre el 17 y el 25\%. Únicamente el estudio de Wong et al. ${ }^{18}$ presentó un porcentaje mucho más elevado, siendo en dicho estudio la lesión más frecuentemente encontrada.

Las lesiones condrales y osteocondrales se hallaron tanto en pacientes con edad avanzada como en más jóvenes, sin encontrar relación respecto a la edad. Esto se diferencia en parte con la serie de Caloia et al. ${ }^{20}$, donde los 2 pacientes de mayor edad (de entre 62 y 75 años) presentaban dichas lesiones. Caloia et al. lo relacionaron con posibles cambios degenerativos en vez de con lesiones agudas.

La incidencia de lesiones de los ligamentos extrínsecos ha sido analizada previamente por diversos estudios. Caloia et al. ${ }^{20}$ encontraron una incidencia del $8 \%(2 / 24)$, similar a la encontrada en nuestro análisis, con una lesión del ligamento radioescafocapitate en su prolongación mediocarpiana $(5,9 \%$,$) . Sin embargo, Jørgsholm et al. { }^{19}$ presentaron una incidencia muy por encima del resto de los estudios, alcanzando incidencias del 22\% (9/41). En todos los estudios, las lesiones fueron parciales. También encontramos la rotura de la cápsula dorsal en un paciente $(5,9 \%)$, solamente superado por los 16 de 41 pacientes (37\%) que observaron Jørgsholm et al. ${ }^{19}$ en su estudio.

Los avances de las técnicas percutáneas de fijación con tornillo y las altas tasas de consolidación obtenidas en los diferentes estudios, además de presentar complicaciones poco frecuentes, han llevado a que cada día se utilicen más la fijación percutánea y la movilización precoz en el tratamiento de estas lesiones ${ }^{7-9}$. Existe controversia sobre si este tipo de lesiones tiene un impacto negativo en los resultados funcionales ${ }^{13,14}$. Sin embargo, si las lesiones asociadas pasan inadvertidas durante la cirugía, podrían llegar a producir inestabilidades sintomáticas del carpo. El daño del cartílago articular en el momento de la lesión es una de las posibles explicaciones para la persistencia de síntomas después de la consolidación de la fractura. En referencia a esta suposición, en nuestra serie, 4 de los 6 pacientes $(66,6 \%)$ que presentaban lesiones condrales en el momento de la artroscopia obtuvieron los resultados menos favorables al valorarlos mediante el Mayo Wrist Score.

Estamos de acuerdo con Jørgsholm et al. ${ }^{19}$ en cuanto a los beneficios y las indicaciones que presenta la artroscopia de muñeca en fracturas desplazadas o no de escafoides para la fijación quirúrgica y la movilización precoz, ya que podría evitar los efectos perjudiciales de las lesiones asociadas que podrían pasar inadvertidas. La ventaja es que la artroscopia permite la evaluación de los diversos ligamentos, que son difíciles de ver con las técnicas de imagen convencionales, y ayuda en la confirmación tanto de la correcta reducción de la fractura, como de la posible extrusión del tornillo tras la osteosíntesis 


\section{Conclusiones}

La artroscopia de muñeca permite diagnosticar y tratar las lesiones asociadas a las fracturas de escafoides carpiano, de forma precoz. Existe una alta incidencia de lesiones asociadas con las fracturas agudas del cuerpo del escafoides, siendo las lesiones parciales del ligamento EL y del CFCT las más frecuentemente asociadas.

\section{Conflicto de intereses}

Los autores declaran no tener ningún conflicto de intereses.

\section{Bibliografía}

1. Gellman H, Caputo RJ, Carter V, et al. Comparison of short and long thumb spica casts for nondisplaced fractures of the carpal scaphoid. J Bone Joint Surg Am. 1989;71:354-7.

2. Kaneshiro SA, Failla JM, Tashman S. Scaphoid fracture displacement with forearm rotation in a short arm thumb spica cast. J Hand Surg. 1989;71:354-7.

3. Rettig AC, Kollias SC. Internal fixation of acute stable scaphoid fractures in the athlete. Am J Sports Med. 1996;24:182-6.

4. Skirven T, Trope J. Complications of immobilization. Hand Clin. 1994;10:53-61.

5. Steinmann SP, Adams JE. Scaphoid fractures and nonunions. J Orthop Sci. 2006;11:424-31.

6. Streli R. Perkutane Verscraubung des Handkahnbeines mit Bohrdrahtkompressionschraube. Zentral bi Chir. 1970;95:1060-78.

7. Slade JF 3rd, Geissler W, Gutow A, Merrell G. Percutaneous internal fixation of selected scaphoid nonunion with an arthroscopically assisted dorsal approach. J Bone Joint Surg Am. 2003;85:20-32.
8. Haddad FS, Goddard NJ. Acute percutaneous scaphoid fixation: A pilot study. J Bone Joint Surg Br. 1998;80:95-9.

9. Jupiter JB, Herndon JH. Acute fractures of the scaphoid. J Am Acad Orthop Surg. 2000;8:225-31.

10. Slade J 3rd, Gutow A, Geissler W. Percutaneous internal fixation of scaphoid fractures via an arthroscopically assisted dorsal approach. J Bone Joint Surg Am. 2002;84:21-37.

11. Ledoux P, Chahidi N, Moermans JP, Kinnen L. Percutaneous Herbert screw osteosynthesis of the scaphoid bone. Acta Orthop Belg. 1995;61:43-7.

12. Whipple TL. Stabilization of the fractured scaphoid under arthroscopy control. Orthop Clin North Am. 1995;26:749-54.

13. Shih JT, Lee HM, Hou YT, Tan CM. Results of arthroscopic reduction and percutaneous fixation for acute displaced scaphoid fractures. Arthroscopy. 2005;5:620-6.

14. Amadio PC, Berquist TH, Smith DK, Ilstrup DM, Cooney WP 3rd, Linscheid RL. Scaphoid malunion. J Hand Surg Am. 1989;14(4):679-87.

15. Geissler WB, Freeland AE. Arthroscopically assisted reduction of intraarticular distal radial fractures. Clin Orthop. 1996;327:125-34.

16. Palmer AK. Triangular fibrocartilage complex lesions: A classification. J Hand Surg. 1989;14A:594-606.

17. Geissler WB. Arthroscopic assisted fixation of fractures of the scaphoid. Atlas Hand Clin. 2003;8:37-56.

18. Wong TC, Yip TH, Wu WC. Carpal ligament injuries with acute scaphoid fractures -A combined wrist injury. J Hand Surg Br. 2005;30:415-8.

19. Jørgsholm P, Thomsen NO, Björkman A, Besjakov J, Abrahamsson SO. The incidence of intrinsic and extrinsic ligament injuries in scaphoid waist fractures. J Hand Surg Am. 2010;35(3):368-74.

20. Caloia MF, Gallino RN, Caloia H, Rivarola H. Incidence of ligamentous and other injuries associated with scaphoid fractures during arthroscopically assisted reduction and percutaneous fixation. Arthroscopy. 2008;24:754-9. 